\title{
Dynamic forces with random amplitudes from rhythmic jumps and squats
}

\author{
Marek Pańtak ${ }^{1 *}$ \\ ${ }^{1}$ Cracow University of Technology, Institute of Building Materials and Engineering Structures, \\ 31-155 Cracow, Warszawska 24, Poland
}

\begin{abstract}
In the paper the load models of the dynamic forces generated during jumps and squats taking into account random changes in the amplitudes of the forces are presented. The models were elaborated on the basis of series of laboratory measurements of continuously and rhythmically performed jumps and squats in frequency range $1.40-3.80 \mathrm{~Hz}$.
\end{abstract}

\section{Introduction}

The ground reaction forces (GRFs) arising during jumps and squats can be an important dynamic load of the structures. These types of dynamic loads may appear on building floors, stadium tribunes, communication galleries, footbridges etc. The GRFs generated during squats and jumps exceeds two to over three times the body weight $G$ of a squatting or jumping person. For this reason, squats or jumps can leads to significant vibrations of the structures, especially in the case of structures with low damping.

Rhythmically and continuously performed jumps and squats lead to the creation of a periodic (or near-periodic) forces presented in Fig. 1.

a)

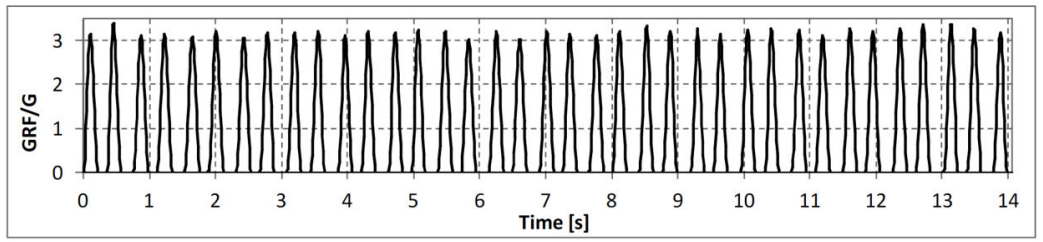

b)

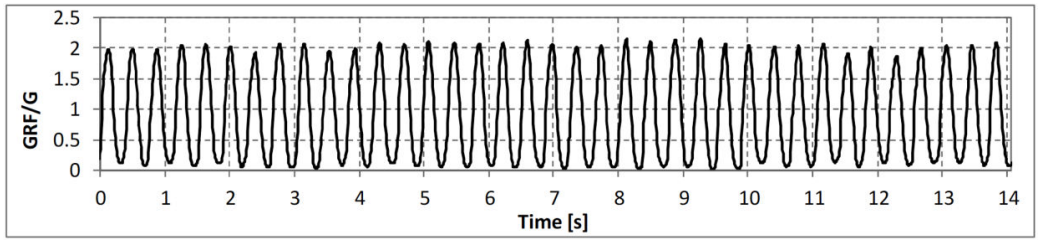

Figure 1. Normalized force $G R F / G$ measured during a) jumps, b) squats with frequency $2.60 \mathrm{~Hz}$ (random amplitudes)

* Corresponding author: mpantak@pk.edu.pl 
These periodic forces can be described by functions presented, inter alia, in $[1,2]$ for jumps (eq. (1)) and in [3, 4] for squats (eq. (2)). Other proposals for GRFs models and additional informations about modelling of forces resulting from different types of human activities can also be found in [5, 6, 7, 8, 9].

The load model of the GRFs generated during jumps presented in [1] and analysed in [2] was described by means of Hann function in the form of equation (1):

$$
F_{j}(t)= \begin{cases}A_{j} \cdot G \cdot\left[0.5-0.5 \cos \left(\frac{2 \pi \cdot t}{t_{c j}}\right)\right] & \text { for } \quad t \leq t_{c j} \\ 0 & \text { for } \quad t_{c j}<t \leq T_{j}\end{cases}
$$

where:

$A_{j}=\frac{2 T_{j}}{t_{c j}}-$ dynamic amplification factor for jumps,

$G$ - body weight of jumping person (usually assumed $G=700-800 \mathrm{~N}$ ),

$T_{j}-$ period of jumps $T_{j}=1 / f_{j}[\mathrm{~s}]$,

$f_{j}$ - frequency of jumps [Hz],

$t_{c j}$ - contact time of the feet with the ground during jumps (Tab. 1),

$t$ - time steep $t \geq 0$.

The mean value of the contact time $t_{c j}$ and a standard deviation of $t_{c j}$ established by author on the basis of the laboratory tests of 5 jumping people (age 19, 25, 35, 40 and 45 years), during jumps in the frequency range $1.40-3.80 \mathrm{~Hz}$ performed in two series of $15 \mathrm{~s}$ for each frequency are presented in Tab. 1. Recommendations from other authors can also be found in [1] and [2].

Table 1. Contact time of the feet with the ground during jumps $t_{c j}$

\begin{tabular}{|l|c|c|c|c|c|c|c|c|}
\hline Frequency $\boldsymbol{f}_{\boldsymbol{j}}[\mathrm{Hz}]$ & $\mathbf{1 . 4 0}$ & $\mathbf{1 . 6 0}$ & $\mathbf{2 . 0 0}$ & $\mathbf{2 . 4 0}$ & $\mathbf{2 . 8 0}$ & $\mathbf{3 . 2 0}$ & $\mathbf{3 . 6 0}$ & $\mathbf{3 . 8 0}$ \\
\hline Contact time $\boldsymbol{t}_{\boldsymbol{c}}[\mathrm{s}]$ & $\begin{array}{c}0.500 \\
\pm 0.028\end{array}$ & $\begin{array}{c}0.403 \\
\pm 0.017\end{array}$ & $\begin{array}{c}0.302 \\
\pm 0.022\end{array}$ & $\begin{array}{c}0.261 \\
\pm 0.009\end{array}$ & $\begin{array}{c}0.237 \\
\pm 0.012\end{array}$ & $\begin{array}{c}0.198 \\
\pm 0.009\end{array}$ & $\begin{array}{c}0.195 \\
\pm 0.008\end{array}$ & $\begin{array}{c}0.191 \\
\pm 0.012\end{array}$ \\
\hline
\end{tabular}

The comparison of the forces generated by means of equation (1) with the GRFs measured during laboratory tests showed that equation (1) applies to the jumping frequency $f_{j} \geq 2.2 \mathrm{~Hz}$ for which the factor $A_{j}$ is in the range $3.0-3.5$. In the case of jumps with frequency $f_{j}=1.40-2.20 \mathrm{~Hz}$ the value of the factor $A_{j}$ is in the range $2.0-3.0$. For the correct application of equation (1) in the frequency range $f_{j}=1.40-2.20 \mathrm{~Hz}$ a correction coefficients for dynamic amplification factor $A_{j}$ should be used. The author's proposals for correction coefficients are presented in Tab. 2.

Table 2. Correction coefficients for eq. (1) in frequency range $1.40 \leq f_{\mathrm{j}} \leq 3.80 \mathrm{~Hz}$

\begin{tabular}{|c|c|c|c|c|c|c|}
\hline Frequency $f_{j}[\mathrm{~Hz}]$ & $\mathbf{1 . 4 0}$ & $\mathbf{1 . 6 0}$ & $\mathbf{1 . 8 0}$ & $\mathbf{2 . 0 0}$ & $\mathbf{2 . 2 0 - 3 . 0 0}$ & $f_{\mathrm{j}}>\mathbf{3 . 0 0}$ \\
\hline$\lambda$ & 0.65 & 0.75 & 0.80 & 0.90 & 1.00 & 1.10 \\
\hline \multicolumn{7}{|c|}{$A_{j}=\lambda \frac{2 T_{j}}{t_{c j}}$} \\
\hline
\end{tabular}


The GRFs generated during squats can be determined using equation (2) appropriate for the frequency of squats $f_{s q}>1.60 \mathrm{~Hz}[3,4]$ :

$$
F_{s q}(t)=G \cdot\left[1.1+\sin \left(2 \pi \cdot f_{s q} \cdot t\right)\right]
$$

where:

$G$ - body weight of squatting person,

$f_{s q}$ - frequency of squats $[\mathrm{Hz}]$,

$t$ - time steep $t \geq 0$.

In Fig. 2 the periodic GRFs determined by means of equations (1) and (2) are presented.

a)

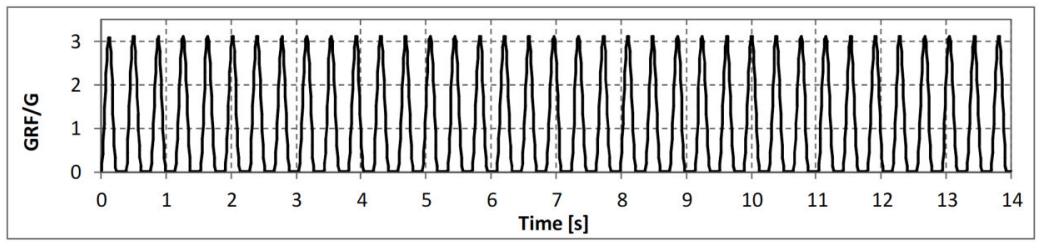

b)

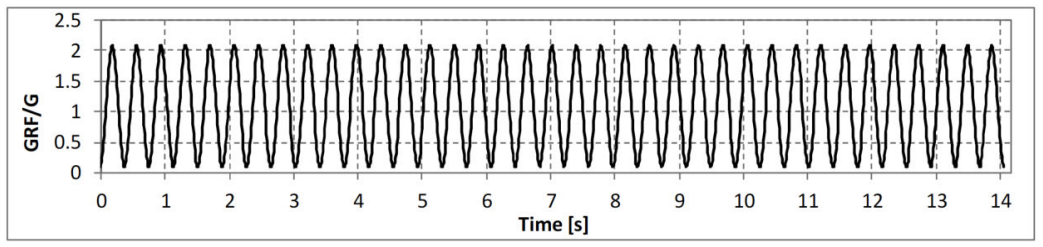

Figure 2. Normalized force $G R F / G$ for a) jumps: eq. (1), b) squats: eq. (2) with frequency $2.60 \mathrm{~Hz}$ (constant amplitudes)

Comparing the courses of GRFs presented in Fig. 1 and Fig. 2 it can be seen that the $G R F$ s amplitudes presented in Fig. 1 slightly change randomly during the rhythmically and continuously performed jumps and squats whereas the GRFs amplitudes presented in Fig. 2 are constant. In order to take into account a random variability of amplitudes in models (1) and (2) the use of a randomly generated force coefficient was proposed.

\section{Random force coefficient}

The random force coefficient was developed using a multiplicative linear congruential generator $(M L C G)$ defined in the general form by the equation (3).

$$
Y_{n}=\left(a \cdot Y_{n-1}\right) \bmod m
$$

where:

$Y_{n}-n$-th pseudorandom number, $n=1,2, \ldots$

$Y_{n-1}$ - previous pseudorandom number $\left(Y_{0}-\right.$ pseudorandom seed),

$a-$ the "multiplier",

$m$ - the "modulus",

$x$ mod $y$ - the remainder of the division of $x$ by $y$ (modulo operation). 
The $M L C G$ allows to generate large sets of random numbers under the condition of proper selection of generator parameters $m, a, Y_{0}$. Various parameters of linear congruential generators with long period, ensuring formal submission of randomness tests, can be found in the literature $[10,11,12,13,14]$. For further analyses, it was adopted $m=2^{35}$, $a=2^{2} \cdot 23^{7}+1, Y_{0}=2^{19}$.

To calculate the remainder of the division of $a \cdot Y_{n-1}$ by $m$ the equation (4) was used.

$$
Y_{n}=\left(a \cdot Y_{n-1}\right)-\left\lfloor\frac{\left(a \cdot Y_{n-1}\right)}{m}\right\rfloor \cdot m
$$

where:

$\left\lfloor\frac{\left(a \cdot Y_{n-1}\right)}{m}\right\rfloor-$ the integer part of the division.

The first two pseudorandom numbers have the form:

$$
Y_{1}=\left(a \cdot Y_{0}\right)-\left\lfloor\frac{\left(a \cdot Y_{0}\right)}{m}\right\rfloor \cdot m ; \quad Y_{2}=\left(a \cdot Y_{1}\right)-\left\lfloor\frac{\left(a \cdot Y_{1}\right)}{m}\right\rfloor \cdot m ; \quad \text { etc. }
$$

The random numbers $Y_{n}$ generated by means of eq. (4) are used in formula (6) proposed for the random force coefficient $\Phi_{F}$ determined in the range from $\left(1-\sigma_{F} / 2\right)$ to $\left(1+\sigma_{F}\right)$.

$$
\Phi_{F}\left(\sigma_{F}\right)=\frac{Y_{n}}{m} \cdot\left[\left(1+\sigma_{F}\right)-\left(1-\frac{\sigma_{F}}{2}\right)\right]+\left(1-\frac{\sigma_{F}}{2}\right)=1+\frac{3 \sigma_{F} Y_{n}}{2 m}-\frac{\sigma_{F}}{2}
$$

where:

$\sigma_{F}-$ standard deviations of the GRFs amplitudes for jumps or squats,

$\left(1-\sigma_{F} / 2\right)-$ lower limit of the coefficient range,

$\left(1+\sigma_{F}\right)-$ upper limit of the coefficient range.

Adoption of the lower limit of the coefficient range as $\left(1-\sigma_{F} / 2\right)$ enables correct calculation of $G R F$ s amplitudes with values lower than the mean value of the GRFs.

The standard deviations $\sigma_{F}$ of the GRFs amplitudes were determined based on the results of jumping and squatting tests in the frequency range $1.40-3.80 \mathrm{~Hz}$ (duration of the test $2 \times 15 \mathrm{~s}=30 \mathrm{~s}$ for each frequency and each person, group size 5 people, age 19, 25, 35, 40 and 45 years, equipment: force measuring platform ZEBRIS FDM-1.5 placed on a rigid floor, software WinFDM, electronic metronome). Determined values of $\sigma_{F}$ are presented in Tab. 3.

Generally the $\sigma_{F}$ for jumps can be assumed in the range of $0.06-0.20$ and for squats in the range of $0.03-0.11$. During the tests and data analyses it was observed that values of $\sigma_{F}$ are relatively low $\left(\sigma_{F}=0.05-0.10\right)$ in situations when during jumps a jumping person bounce and land on the fore parts of the feet ("soft/gentle jumps"). In the case when jumping person bounce and land on the entire surfaces of the feet ("hard jumps") values of $\sigma_{F}$ are relatively high $\left(\sigma_{F}=0.10-0.20\right)$. Moreover, the GRFs amplitudes in the case of "hard jumps" are greater by about $15-25 \%$ than the amplitudes of "soft jumps". Furthermore, it must be noted that people performing squats and jumps during the tests were very well synchronized with the metronome indicating the pace of movement. Similar situation can occur in the case of synchronization of a jumping/squatting person with a vibrating structure. The values of $\sigma_{F}$ will be higher if the person performing jumps or squats does not feel/hear any impulse indicating the pace of movement. 
Table 3. The values of standard deviations of $G R F$ s amplitudes for jumps and squats in the frequency range $1.40 \leq f_{\mathrm{j}} \leq 3.80 \mathrm{~Hz}$

\begin{tabular}{|c|c|c|}
\hline \multirow{2}{*}{$\begin{array}{c}\text { Frequency } \\
{[\mathbf{H z}]}\end{array}$} & \multicolumn{2}{|c|}{ Standard deviation $\boldsymbol{\sigma}_{\boldsymbol{F}}$} \\
\cline { 2 - 3 } & Jumps $\boldsymbol{\sigma}_{\boldsymbol{F}, \boldsymbol{j}}$ & Squats $\boldsymbol{\sigma}_{F, s \boldsymbol{q}}$ \\
\hline $\mathbf{1 . 4 0}$ & $0.09-0.17$ & $0.03-0.06$ \\
\hline $\mathbf{1 . 6 0}$ & $0.10-0.19$ & $0.04-0.06$ \\
\hline $\mathbf{1 . 8 0}$ & $0.06-0.15$ & $0.03-0.07$ \\
\hline $\mathbf{2 . 0 0}$ & $0.09-0.18$ & $0.04-0.07$ \\
\hline $\mathbf{2 . 2 0}$ & $0.12-0.19$ & $0.03-0.07$ \\
\hline $\mathbf{2 . 4 0}$ & $0.10-0.20$ & $0.03-0.06$ \\
\hline $\mathbf{2 . 6 0}$ & $0.10-0.19$ & $0.04-0.08$ \\
\hline $\mathbf{2 . 8 0}$ & $0.06-0.16$ & $0.04-0.08$ \\
\hline $\mathbf{3 . 0 0}$ & $0.07-0.15$ & $0.04-0.09$ \\
\hline $\mathbf{3 . 2 0}$ & $0.08-0.18$ & $0.04-0.08$ \\
\hline $\mathbf{3 . 4 0}$ & $0.11-0.21$ & $0.05-0.09$ \\
\hline $\mathbf{3 . 6 0}$ & $0.11-0.19$ & $0.06-0.11$ \\
\hline $\mathbf{3 . 8 0}$ & $0.11-0.19$ & $0.06-0.09$ \\
\hline
\end{tabular}

Ultimately the equations describing the GRFs generated during jumps and squats with random values of amplitudes can be written in the form of equations (7).

$$
F_{\Phi, j}=\Phi_{F}\left(\sigma_{F, j}\right) \cdot F_{j}(t) \quad \text { and } \quad F_{\Phi, s q}=\Phi_{F}\left(\sigma_{F, s q}\right) \cdot F_{s q}(t)
$$

Implementation of the random force coefficient $\Phi_{F}$ in analyses require generation of the single impulse of the $G R F$ for jumps or squats in the time range $0 \leq \mathrm{t} \leq T$ ( $T$ - period of jumps or squats) and duplication of the force impulse multiplied by random coefficient $\Phi_{F}$.

The exemplary graphs of the GRFs for jumps and squats generated using the random force coefficients $\Phi_{F, j}$ and $\Phi_{F, s q}$ are shown in Fig. 3 .

a)

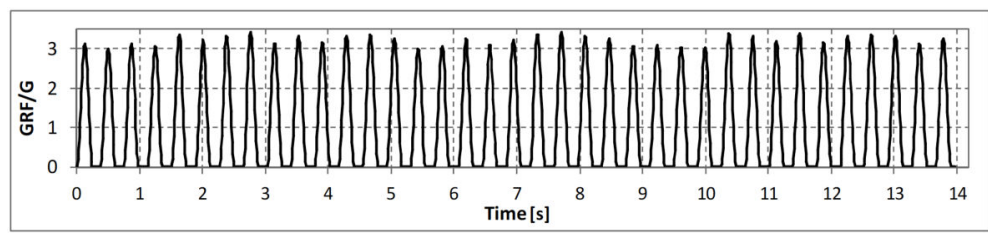

b)

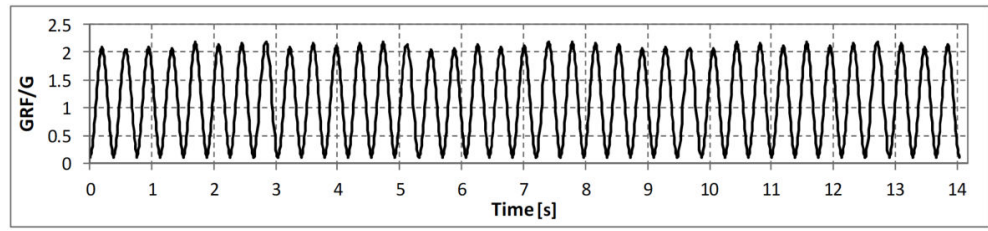

Figure 3. Normalized force $G R F / G$ generated during a) jumping with frequency $2.60 \mathrm{~Hz}$ for $\left.\sigma_{F, j}=0.10, \mathrm{~b}\right)$ squatting with frequency $2.60 \mathrm{~Hz}$ for $\sigma_{F, s q}=0.05$ 


\section{Summary}

The paper presents the proposal of application of random force coefficient for description of random changes of the GRFs amplitudes generated during jumps and squats. The force coefficient was defined with the use of multiplicative linear congruential generator of pseudorandom numbers. A simple methodology of generation of random GRFs amplitudes enables easy implementation of the proposal in dynamic analyses.

The maximum normalized amplitude $G R F / G$ generated for jumps (eq. (1)) with frequency of $2.6 \mathrm{~Hz}$ reaches the value of 3.13 (Fig. 2a). Normalized GRFs amplitudes obtained during laboratory tests (Fig. 1a) change from 3.00 to 3.38. Normalized amplitudes generated taking into account random force coefficient (for $\sigma_{F, j}=0.10$ ) change from 2.98 to 3.42 (Fig. 3a). In the case of squats with frequency $2.6 \mathrm{~Hz}$ the normalized amplitude generated by equation (2) reaches the value of 2.10 (Fig. 2b). The amplitude obtained during laboratory tests presented in Fig. $1 \mathrm{~b}$ change from 1.88 to 2.16 . Normalized amplitudes generated taking into account random force coefficient (for $\sigma_{F, s q}=0.05$ ) change from 2.00 to 2.20 .

It may be noted that the GRFs amplitudes generated using the random force coefficient $\Phi_{F}$ obtained for jumps are comparable with laboratory tests results. In the case of squats the random GRFs amplitudes are slightly higher than amplitudes from laboratory tests. It should be noted that this result strongly depends on the level of synchronisation of the person performing squats with impulse indicating the pace of movement (metronome or vibrating surface/structure). In the case of good synchronization of the person with the impulse of pace the accuracy of determination of the $G R F$ random amplitudes increases (compare Fig. $1 \mathrm{~b}$ for $t=4.0-11.0 \mathrm{~s}(G R F / G=2.00-2.16)$ and Fig. 2b).

Appropriate assumption of the $\sigma_{F}$ value allows for more accurate simulation of the $G R F$ s values and in consequence a more accurate simulation of the dynamic response of the structure. However, the value of $\sigma_{F}$ should be assumed within a reasonable range.

Further researches may concern the study of the effect of structure vibrations (dynamic susceptibility of the structure) on the values and distribution of the GRFs amplitudes.

\section{References}

[1] R. G. Faísca, Ph.D. Thesis, Univ. Federal do Rio de Janeiro (2003)

[2] E. Caetano, A. Cunha, C. Moutinho, J. Bridge Eng. 16, 375-382 (2011)

[3] M. Pańtak, Proc. Int. Conf. Footbridge 2017 (2017)

[4] M. Pańtak, IOP Conf. Ser.: Mater. Sci. Eng. 245 (2017)

[5] K. Żółtowski Pedestrian on footbridges - loads and the dynamic response of the structure, Monograph 82 (GUT Publishing House, Gdańsk, 2007) - in Polish

[6] K. Żółtowski Proc. Int. Conf. Footbridge 2008 (2008)

[7] S. Živanović, A. Pavic, P. Reynolds, J. Sound and Vib. 279(1-2), 1-74 (2005)

[8] P. Hawryszków, Proc. Int. Conf. Footbridge 2011 (2011)

[9] P. Hawryszków, Proc. $9^{\text {th }}$ Int. Conf. on Struct. Dyn., EURODYN 2014 (2014)

[10] J.E. Gentle, Random Number Generation and Monte Carlo Methods (Springer, 2003)

[11]R. Wieczorkowski, R. Zieliński, Computer random number generators (WNT, Warsaw, 1997) - in Polish

[12]P. Bratley, B.L. Fox, L.E. Schrage, A Guide to Simulation (Springer, 1987)

[13]B.D. Ripley, J. Comp. and App. Math. 31, 153-163 (1990)

[14] G.S. Fishman, Math. Comp. 54, 331-344, (1990) 